\title{
Review
}

\section{Research Progress on Infectious Inflammation Markers in Blood}

\author{
Xifeng Dong \\ Department of Hematology-Oncology, Tianjin Medical University General Hospital, Tianjin, China
}

\author{
Keywords \\ infection, inflammation, acute phase protein, \\ enzyme and test \\ Correspondence \\ Xifeng Dong, \\ E-mail: dongxifeng@sdu.edu.cn \\ DOI: $10.1515 / \mathrm{ii}-2017-0112$
}

\begin{abstract}
Acute phase protein (APP) is a type of special protein closely related to infection inflammation. In recent years, a large number of studies have shown that multiple positive and negative APPs, including C-reactive protein, serum amyloid A, procalcitonin, haptoglobin, alphal acid glycoprotein, ceruloplasmin, fibrinogen, prealbumin, leptin, albumin, and plasma fibronectin, are significantly correlated to infectious inflammation and that this method is more accurate and reliable than somatic cell test, erythrocyte sedimentation rate test, enzyme activity and content change test, and the like. Therefore, APP could be used as an infectious inflammation marker.
\end{abstract}

Inflammation is a defensive response to external stimuli. It usually manifests as redness, swelling, fever, pain, dysfunction, and other local symptoms. In general, inflammation could be divided into infectious inflammation caused by bacterial or viral infection and non-infectious inflammation caused by immune system defects and other non-infectious factors.

When a living organism is infected with bacteria, the living organism usually suffers from fever, white blood cell count increase (neutrophil count increase), erythrocyte sedimentation rate (ESR) increase, increase of multiple acute phase proteins (APPs), and other symptoms ${ }^{[1]}$. At present, diagnosis and treatment of infectious diseases depend on traditional methods, such as white blood cell count, classification, and ESR, in most cases. However, these indicators all have their limitations and uncertainties as white blood cell count and ESR increase involve multiple factors. For example, pregnancy, strenuous exercises, intoxication, acute hemorrhage, and other non-infectious factors cause white blood cell count increase. In addition, ESR is influenced by factors irrelevant to infection (nature of red blood cells, serum protein, lipid level, age, sex, anemia, and the like). In addition, ESR slowly reacts to acute inflammation (it usually starts to increase in 2 days to 3 days). ESR does not decrease to normal level despite several weeks of treatment ${ }^{[3]}$. Therefore, detecting reliable sensitive indicators for inflammation diagnosis and making a timely judgment to provide patients with timely treatment is highly important.

In recent years, in-depth studies have been conducted on infectious inflammation and people have discovered many sensitive factors influenced by infectious inflammation, of which enzymes are one of the factors. Basing on relevant studies, the researchers found that a living organism showing inflammatory responses suffers from significant changes of specific enzymes from within, such as lactic dehydrogenase, a1-antitrypsin, aspartate amino transferase, catalase, alkaline phosphatase ${ }^{[4]}$, phosphokinase, xanthine oxidase, fibrinolysin, lactoperoxidase, lipase, and the like. Therefore, enzymes are taken as a detection marker for infectious inflammation in many studies ${ }^{[5]}$. However, enzymes are also influenced by multiple factors. For example, temperature, time, $\mathrm{pH}$, ion concentration in the medium, substrate, and the like all cause enzyme content and activity changes. Therefore, the use of enzyme activity as a diagnosis indicator might sometimes be unreliable.

Another important type of inflammation indicator is the APP. APP is mainly synthesized by hepatic cells, but 
it could also be synthesized by the reticuloendothelial system, neutrophil granulocytes, and vascular endothelial cells. When the organism displays stress reactions during infection, trauma, or other diseases, the content of these substances in the blood changes. An increase in concentration indicates positive APP, while a decrease indicates negative APP. APP is not influenced by fever, ESR increase, white blood cell count increase, or the like. In addition, APP measurement is not influenced by antibiotics, immunosuppressors, or hormones ${ }^{[6]}$. Therefore, it could be used as a reliable inflammation symptom indicator and is significant for the identification of tissue inflammation and necrosis.

\section{Positive APP}

\section{C-reactive protein}

C-reactive protein (CRP) is a typical PPA synthesized by the liver. It is present in small amounts in normal serum. In the serum of a patient in the acute phase of inflammation or with malignant tumor, local anemia, tissue damage, or the like, CRP content increases a thousand-fold. After $4 \mathrm{~h}$ to $6 \mathrm{~h}$ from the initial inflammation stage, it immediately increases and reaches a peak in $36 \mathrm{~h}$ to $50 \mathrm{~h}$. Once the infection is effectively controlled, it quickly decreases to the normal level. Zhu et al. ${ }^{[7]}$ found that only bacterial infection causes an increase in CRP level. When CRP exceeds 140 $\mathrm{mg} / \mathrm{L}$, bacterial infection certainly exists. In addition, CRP level is positively correlated to severity of infection. In viral infection, however, CRP level usually does not increase (except for infections involving adenovirus and herpes virus). For diagnosis of acute infectious diseases, detection of serum CRP is more accurate, reliable, and sensitive than detection of white blood cell count ${ }^{[9]}$. Besides, CRP is unlike IL-6 and other cytokines, whose concentrations fluctuate with day and night shifts ${ }^{[10]}$.

\section{Serum amyloid $A$}

Serum amyloid A (SAA), a heterogeneous protein, is synthesized by activated macrophages and fibroblasts in the liver. It has an extremely short half-life of approximately 50 min. Dai et al. ${ }^{[11]}$ conducted a research on children with infectious diseases showing that the SAA level of the bacteria-infected group in the acute stage is significantly higher than that of the normal control group and the virusinfected group and that the serum SAA level of the virusinfected group in the acute stage is significantly higher than that of the normal control group. A research by Miwata et al. ${ }^{[12]}$ indicates that in children, SAA increases during the acute stage of virus infection and decreases to the normal level during the recovery stage. In chronic viral infection, however, SAA does not increase. In addition, SAA is positively correlated to CRP on a large scale. SAA increases more significantly than CRP. SAA might be a more useful influenza virus infection marker than CRP ${ }^{[13]}$.

\section{Procalcitonin}

Procalcitonin (PCT), the precursor of calcitonin, is a glucoprotein with a half-life $25 \mathrm{~h}$ to $30 \mathrm{~h}$ and high in vitro and in vivo stability. It is produced by $\mathrm{C}$ cells of the thyroid gland under physiological conditions but is present in very small amounts (less than $0.1 \mu \mathrm{g} / \mathrm{L}$ ). When the amount exceeds 0.5 $\mu \mathrm{g} / \mathrm{L}$, it is considered abnormal. In a research on PCT by Balci et al. ${ }^{[14]}$, PCT is found to be a diagnostic indicator in the early stage of bacterial or fungal infection; they also found that PCT increases only during infection and usually does not increase when no infection is involved and found that the increased value is closely related to severity of infection. $\mathrm{Lu}^{[15]}$ analyzed bacterial and viral infections and found that serum PCT level significantly increases in bacterial infection but not in viral infection. In addition, basing on foreign studies ${ }^{[16]}$, PCT increases faster than CRP. In local infection, PCT usually does not increase but CRP increases ${ }^{[17]}$. This finding indicates that CRP might be an important indicator for non-systemic infection. In systemic infection, PCT might be a reliable indicator.

\section{Haptoglobin}

Haptoglobin (HP) is an APP in human serum that can combine with free hemoglobin. Basing on some studies ${ }^{[18]}$, the researchers found that the most sensitive APP of a cow combines with globin and serum amyloid protein. Their contents significantly increase during acute inflammation by approximately 100 times. Jiao et al. ${ }^{[19]}$ vaccinated cows against Staphylococcus aureus through a papillary duct and found that both contents increase significantly in the mild sample at 2 and 8 days. 


\section{Alpha-1 acid glycoprotein}

Alpha-1 acid glycoprotein (AAG) is mainly produced by hepatic macrophases and granulocytes. It is present in low content in the normal human body and is considered a sensitive indicator reflecting inflammation activities or the acute stage. In a research on AAG in patients' blood, Zhang ${ }^{[20]}$ reported that in children with infectious diseases, AAG level of children in the group with bacterial infection significantly increased compared with the normal control group and the group with viral infection. No significant differences were found between the AAG of the group with viral infection and the AAG of the normal control group, and CRP is positively correlated to AAG in the group with bacterial infection. Another research indicated that AAG significantly increased during chronic mammary gland infection.

\section{Ceruloplasmin}

Ceruloplasmin (CP) is synthesized in the liver. CP content in newborn blood is rather low. It gradually increases and reaches the level of an adult at the age of 12 . It has iron oxidase activity and is able to remove superoxide radicals in the body and inhibits lipid oxidation. In infective acute inflammation, CP immediately increases from $2 \mathrm{~h}$ to $24 \mathrm{~h}$ and reaches the maximum value from $72 \mathrm{~h}$ to $96 \mathrm{~h}$. CP, as an APP, significantly increases during infection, myocardial infarction, and other inflammatory diseases ${ }^{[21]}$.

\section{Fibrinogen}

Fibrinogen (Fg), synthesized by parenchyma cells in the liver, has a half-life of 3 to 4 days. It is a blood coagulation protein with the highest blood coagulation factor content, entering the blood circulation after it is produced. Liu et al. ${ }^{[22]}$ indicated that plasma fibrinogen content increases to varying degrees during acute inflammation, cardiovascular disease, and malignant tumor.

\section{Negative APP}

\section{Prealbumin}

Prealbumin (PA), synthesized by hepatic cells, is a nonspecific host defense substance. Its half-life is as short as $12 \mathrm{~h}$. PA concentration in serum quickly decreases in acute infection, which is more obvious than in bacterial infection.
Zhao et al. ${ }^{[23]}$ investigated PA of 30 patients with bacterial infection and 26 patients with viral infection, concluding that PA content decreases during acute bacterial infection. To some degree, this phenomenon is related to the severity of infection, and PA content does not significantly decrease in virus-infected and normal control groups.

\section{Serum leptin}

Leptin is a peptide hormone secreted by adipose cells. Malnourished patients usually have low serum leptin level. Lack of serum leptin increases the body's susceptibility to infection and inflammatory stimulation. Cheng et al. [24] tested the leptin level of children with bacterial infection using radioimmunoassay. Their results indicate that serum leptin level of children in the group with severe infection is significantly lower than that in the group with ordinary infection and that serum leptin level in the acute stage is negatively correlated to TNF- $\alpha$.

\section{Albumin}

Albumin (Alb) is synthesized by parenchymal hepatic cells. The half-life of Alb in serum is approximately 15 days to 19 days. Alb is a protein with the highest content in serum. Zhao et al. ${ }^{[25]}$ conducted a toxicity attack test on chicken with infectious bronchitis virus M41 and then tested the changes in its biochemical serum indicators. The results indicate that Alb content in chicken serum of the group toxicity attack test group significantly decreased.

\section{Plasma fibronectin}

Plasma fibronectin (P-FN), a nonspecific regulin, plays an important role in tissue repair, immunoregulation, body anti-infection activity, and other activities. Zhang et al. ${ }^{[26]}$ tested the P-FN content of 54 infected newborn babies with rocket immunoelectrophoresis and found that P-FN content of newborn babies with infectious diseases in the infection stage is significantly lower than that of the healthy group and that P-FN content gradually increases in the recovery stage.

\section{Conclusion}

In conclusion, inflammation marker (especially APP) test plays a positive guiding role in observation, diagnosis, and treatment of many infectious diseases. At present, in 
correlation studies between individuals with inflammation, only a small number of independent indicators are tested and systematic and comprehensive tests are seldom performed and changes and correlation between various inflammationsensitive factors are seldom analyzed. Therefore, monitoring and dynamic observation of multiple main markers, detection, and relevant clinical practice are predicted to be helpful for the monitoring of disease progression and pathogenesis and would facilitate the understanding of the efficacy of antibiotics and other anti-inflammatory drugs for relevant diseases.

\section{Declarations}

\section{Acknowledgements}

No.

\section{Competing interests}

The author declares that she has no competing interest.

\section{Authors' contributions}

XF Dong made the literature analysis and wrote, discussed and revised the manuscript of this review.

\section{References}

1 Zhan Y, Li J, Pan Y. Application of serum C-reactive protein test in diagnosis of pediatric diseases. Laboratory medicine, 2006, 21 (3):1161

2 Tang X, Wang Q, Wang Y. Value of APRP and white blood cell count of 482 patients with acute infection in test and diagnosis. Clinical education of general practice, 2007, 5(5):397-398.

3 Wang Y. Ning Y, Wu F. Application of serum C-reactive protein test in identification of bacterial infection and viral infection. Journal of modern laboratory medicine, 2007, 22(6):127.

4 Babaei H, Mansouri-Najand L, Molaei M M, et al. A ssesscment of lactate dehydrogenase, alkaline phosphatase and aspartate aminotransferase activit ies in cow's milk as an indicat or of subclinical mastitis.Vet Res Commun, 2007, 31(4):419- 425

5 Gao S, Wang G, Shao Z, et al. An exploration on hazards and detection methods of mastitis milk of cows. China dairy industry, 2008 , $36(2): 58-64$

6 Shen B, Value of C-reactive protein in diagnosis and treatment of newborn babies with infectious diseases. Journal of practical medical techniques, 2008, 15(19): 2497-2498.

7 Zhu J, Quyyumi AA, Norman JE, et al. Cytomegalovirus in the pathogenesis of atherosclerosis: the role of inflammation as reflected by elevated C-reactive protein levels. J Am Coll Cardiol, 1999, 34 (6):1738-1743.

8 Wang Y, Duan L, Wang Z, et al. Significance of C-reactive protein test in infectious diseases. People's military surgeon, 2007, 50 (7):419-420.

9 Wang P. Clinical value of C-reactive protein test for infectious diseases of the lower respiratory tract. Journal of clinical and experimental medicine, $2006,4(4): 363-364$

10 Ewart H K M, Rider P M, Rif ai N, et al. Absence of diu rnal variati on of C-reactive prot ein levels in healthy human subject s. Clin $\mathrm{Ch}$ em, 2001, 47:426-430

11 Dai L, Shen S, Wu J, et al. Value of joint detection of SAA and CRP for identification and diagnosis of pediatric infectious diseases in the early stage. Shanghai journal of medical laboratory sciences, 2003, $18(4): 227-228$

12 Miwata H, Yamada T, Okada M, et al. Serum amyloid A protein in acute viral infect ions. Arch Dis Child, 1993, 68 (2):210-214.

13 Wang H, Li M. A comparison of C-reactive protein and S erumamyloid A changes for children with influenza virus infection. China medical herald, 2008, 5(2):43

14 Balci C, Sunguu rtekin H, Gurses E, et al. Usefulness of procalcitonin for diagnosis of sepsis in the intensive care unit. Crit Care, 2003, 7:85-90.

15 Lu Y. Clinical significance of serum PCT and C-reactive protein test in infectious diseases. Journal of Shenyang Medical College, 2006, $8(2): 114-115$

16 Monneret G, Labaune J M, Isaac C , et al. Procalcitonin and C-raective protein levels in neonatal infections. Act a Paediatr, 1997, 86:209-212.

17 Shan $\mathrm{H}$, Zhang W, Chu S. A new indicator for detection of severe systemic infection-PCT. Shanghai medical journal, 2001, 24(11):699-701.

18 Donaszi IA, Scharekp P, Falus A, et al. Hepaticacute-phasereaction in hist amine-deficient gene targeted mice. Inflammopharmacology, 2004, 12(1):47-55.

19 Jiao L, Wang J, Ma J, et al. An exploration on sensitive indicators for staphylococcus aureus cow mastitis test. Chinese journal of veterinary medicine, 2006, 42(5):31.

20 Zhang J, Yi Z, Luo X, et al. Application of serum C-reactive protein, $\alpha 1$ acidoglycoprotein and prealbumin detection in infectious diseases of children. Clinical Medicine of China, 2006, 22(5): 470-475.

21 Zhang M. Serum ceruloplasmin detection in gynecological diseases and and its clinical significance. Experimental and laboratory medicine, 2008, 26(1):78

22 Liu J, Wang C. Application value of plasma fibrinogen level rise in clinical practice. Laboratory medicine education, 2008, 15(1):44-46.

23 Zhao P, Zhang L. Application of serum prealbumin and C-reactive protein tests in infectious diseases. Clinical auxiliary examination, 2005(7):45 
24 Chen Y, Chang J, Li Y, et al. Serum leptin and tumor necrosis factor changes of children with bacterial infectious diseases and relevant significance. Shangdong Medical Journal. 2004, 44(19):6-7.

25 Zhao X, Su J, Li X, et al. Influence of avian infectious bronchitis virus on chichen serum biochemical indicators. Anhui Agricultural Sciences.
2007, 35(11) 3267-3268.

26 Zhang Y, Yuan X, Yan H. Clinical significance of plasma fibronectin changes in newborn infection. Central Plains Medical Journal. 2004, 31(4):3-4. 